\title{
PSEUDO-MATCHINGS OF A BIPARTITE GRAPH
}

\author{
ALAN BRACE AND D. E. DAYKIN
}

Abstract. Let $G$ be a graph whose edges $(x, y)$ have $x \in X$, $y \in Y,|X|=|Y|<\infty$. A $(t, u)$ cover of $G$ is a set of $t$ edges which cover $\geqq u$ vertices in both $X$ and $Y$. We give conditions on the valency (minimum local degree) and the number of edges which ensure a $(t, u)$ cover or that a Hamiltonian circuit exists.

1. Introduction. Let $X, Y$ be two finite sets each with $s$ elements. Also let $E$ be a set of ordered pairs $(x, y)$ with $x \in X, y \in Y$. Thus we have a bipartite graph $G$ whose vertices are $X \cup Y$ and edges are $E$. By a $(t, u)$ cover $C$ of $G$ we mean a set of $t$ edges of $E$ which between them contain at least $u$ elements of $X$ and of $Y$, so

$$
t=|C| \text { and } u \leqq\left|\bigcup_{(x, y) \in C} x\right| \text { and } u \leqq\left|\bigcup_{(x, y) \in C} y\right| \text {. }
$$

The term pseudo-matching in the title of this note refers to a $(t, u)$ cover and was chosen because a $(t, u)$ cover is a matching when $s=t=u$. Throughout this note we will assume, for obvious reasons, that

$$
1 \leqq u \leqq s, \quad u \leqq t \leqq|E| \leqq s^{2}, \quad 0 \leqq v \leqq s,
$$

where $v$ denotes the valency of $G$, that is, the minimum local degree. Our object is to find the best possible conditions on the valency $v$ and the number $|E|$ of edges of $G$ which ensure that $G$ has a $(t, u)$ cover or a Hamiltonian circuit. We now describe our results.

THEOREM 1. There is $a(t, u)$ cover if $v \geqq 1$ and $v \geqq u-\frac{1}{2} t$.

THEOREM 2. If there is no $(t, u)$ cover then either

(i) $|E| \leqq s(u-1)$ and $v=0$, or

(ii) $|E| \leqq(s-v)(2 u-t-1)+v^{2}$ and $2 \leqq 2 v<u \leqq t<2(u-v)$.

By putting $s=t=u$ in Theorem 2 we obtain the following new criterion for the existence of a matching

Received by the editors February 14, 1972 and, in revised form, February 6, 1973. AMS (MOS) subject classifications (1970). Primary 05A05.

Key words and phrases. Circuit, graph, Hall theorem, Hamiltonian, König, matching, number of edges, Ore, valency.

(c) American Mathematical Society 1974 
THEOREM $2^{\prime}$. If $v \geqq \frac{1}{2} s$ or if $|E|>(s-v)(s-1)+v^{2}$ and $0<v<\frac{1}{2} s$ then $G$ has a matching.

We illustrate possible applications of Theorems 1 and 2 by an example. Each of the spanners in a tool bag has one end to fit a British size nut in the range $B_{1}, \cdots, B_{10}$ and the other end to fit a Continental size nut in the range $C_{1}, \cdots, C_{10}$. If there are at least 2 spanners for each size nut, then an engineer can choose 6 which will between them fit at least half of both the $B_{i}$ and the $C_{i}$ sizes. Also he can choose 4 having no size in common. Moreover if there are 37 spanners in the bag he can choose 51 which will between them fit all the nut sizes.

THEOREM 3. If $2 \leqq v \leqq \frac{1}{2} s$ then $G$ has a circuit of length at least $2 v$.

THEOREM 4. If $v>\frac{1}{2} s$ or if $|E|>(s-v) s+v^{2}$ and $2 \leqq v \leqq \frac{1}{2} s$ then $G$ is Hamiltonian.

To compare these results with those for general graphs see [3]. We will use the following classical result of Hall, König and Ore [2, p. 98].

THEOREM 5. There is $a(u, u)$ cover if $|A| \leqq s-u+|\Gamma A|$ for all $A \subseteq X$.

Here $\Gamma A$ denotes the set $\{y \mid \exists x \in A,(x, y) \in E\}$.

In [1] generalised graphs were considered. Instead of the edges being ordered pairs $(x, y)$ they are ordered $n$-tuples $\left(x_{1}, x_{2}, \cdots, x_{r}\right)$ with $r \geqq 2$ and $x_{i} \in X$. The simplest result proved in [1] is the following. If $|E|>(s-1) s^{r-1}$ then there are $s$ edges in $E$ such that for every $x \in X$ and $i$ in $1 \leqq i \leqq r$ the element $x$ is the $i$ th component of one of the $s$ edges.

2. The valency problem. Assume we have a $(t, u)$ cover. Then if $t \leqq|E|-1$ there is trivially a $(t+1, u)$ cover, while if $t \leqq|E|-2$ and $u \leqq s-1$ and $1 \leqq v$ there is a $(t+2, u+1)$ cover. We will use these ideas repeatedly, and they immediately give us part (i) of our first Lemma. There and elsewhere $m$ denotes the maximum integer $u$ for which $G$ has a $(u, u)$ cover.

LEMMA 1. (i) If $1 \leqq v$ and $u<u^{\prime} \leqq s$ and there is $a(t, u)$ cover then there is $a\left(w, u^{\prime}\right)$ cover where $w=\min \left(|E|, t+2\left(u^{\prime}-u\right)\right)$.

(ii) If there is $a(t, u)$ cover then $m \geqq 2 u-t$.

(iii) If $1 \leqq v$ the least integer $t$ for which there is a $(t, u)$ cover is $t=$ $\max (u, 2 u-m)$.

Proof. Part (ii). Imagine $G$ consists of the $(t, u)$ cover alone and $s=u$. If $A \subset X$ then the $(t, u)$ cover must have an edge for each $x \in A$ and $y \in Y \backslash \Gamma A$. Hence $|A|+u-|\Gamma A| \leqq t$ and the result follows by Theorem 5 . 
Part (iii). If there is to be a $(t, u)$ cover we must have $t \geqq u$. Also by part (ii) we must have $t \geqq 2 u-m$. It is easy to get a $(t, u)$ cover from the $(m, m)$ cover, so the proof is complete.

LEMMA 2. (i) If $1 \leqq v<\frac{1}{2}$ s then $G$ has $a(2 v, 2 v)$ cover.

(ii) If $v \geqq \frac{1}{2} s$ then $G$ has an $(s, s)$ cover (and hence $a(u, u)$ cover for $1 \leqq u \leqq s)$.

Proof. By Theorem 5, if (i) is false, there is a set $A \subseteq X$ with $|A|>$ $s-2 v+|\Gamma A|$. Then $s \geqq|A|>|\Gamma A| \geqq 0$ so $A$ is nonempty, $|\Gamma A| \geqq v$, and there is a $y \in Y \mid \Gamma A$. The valency $v(y)$ of this element $y$ satisfies $v \leqq v(y) \leqq$ $|X \backslash A|=s-|A|<2 v-|\Gamma A| \leqq v$. This contradiction proves (i). If (ii) is false, then as before there is a set $A \subseteq X$ with $|A|>|\Gamma A|$. So $\frac{1}{2} s \leqq v \leqq|\Gamma A|<$ $|A| \leqq s$ and there is a $y \in Y \backslash \Gamma(A)$ with $v \leqq v(y) \leqq|X \backslash A|<\frac{1}{2} s \leqq v$. This second contradiction completes the proof.

ProOf OF THEOREM 1. If $v \geqq \frac{1}{2} s$ the result comes from Lemma 2(ii), so we assume $1 \leqq v<\frac{1}{2} s$. Then there is a $(2 v, 2 v)$ cover by Lemma $2(\mathrm{i})$. If $2 v \geqq u$ we are through, so we assume $2 v<u$. Then by Lemma 1(i) we get a $(2(u-v), u)$ cover, and this yields our desired $(t, u)$ cover because $2(u-v) \leqq t$ by hypothesis.

An example which shows that Theorem 1 and Lemma 2(i) are the best possible is the following. Let $X^{\prime} \subseteq X, Y^{\prime} \subseteq Y,\left|X^{\prime}\right|=\left|Y^{\prime}\right|=s-v$ with $1 \leqq v<\frac{1}{2} s$, and let $G$ contain all possible edges except those of the form $\left(x^{\prime}, y^{\prime}\right)$ with $x^{\prime} \in X^{\prime}, y^{\prime} \in Y^{\prime}$. Hence $m=2 v$ and Lemma 1 (iii) applies.

\section{The number of edges problem.}

LEMMA 3. If $G$ has no $(u, u)$ cover then $2 v<u$ and $|E| \leqq(s-v)(u-1)+v^{2}$.

Proof. We must have $2 v<u$ by Lemma 2(i). By Theorem 5 there is a set $A \subseteq X$ with $s \geqq|A|>s-u+|\Gamma A| \geqq s-u \geqq 0$. So $A$ is not empty and $v \leqq|\Gamma A|<u \leqq s$. Taking $y \in Y \backslash \Gamma A$ we get $v \leqq v(y) \leqq|X \backslash A|=s-|A|$ and hence

$$
v \leqq|\Gamma A| \leqq u-v-1
$$

Now

$$
|E| \leqq s^{2}-|A|(s-|\Gamma A|) \leqq s^{2}-(s-u+|\Gamma A|+1)(s-|\Gamma A|)
$$

which is quadratic in $|\Gamma A|$, with the coefficient of $|\Gamma A|$ positive, and hence takes its maximum value at one or other of the bounds in (2). In fact both bounds give the value in the statement of the lemma, and so the lemma is proved.

PrOOF OF THEOREM 2. We must have $2 v<u$, for otherwise by Lemma 3 there is a $(u, u)$ cover, and hence a $(t, u)$ cover. If $v=0$ and $|E|>s(u-1)$ then again by Lemma 3 there is a $(u, u)$ cover. In a moment we will give 
an example with $v=0,|E|=s(u-1)$ and no $(t, u)$ cover, so that we cannot say more in the $v=0$ case. Suppose now that $2 \leqq 2 v<u$ and $t<2(u-v)$. Another example will show that we cannot say anything unless $|E|>$ $(s-v)(2 u-t-1)+v^{2}$. When this holds there is a $(2 u-t, 2 u-t)$ cover by Lemma 3 , and hence a $(t, u)$ cover by Lemma 1 (i). Our last case is $2 \leqq$ $2 v<u$ and $t \geqq 2(u-v)$. Here $1 \leqq v<\frac{1}{2} s$ because $u \leqq s$ and so we get a $(2 v, 2 v)$ cover from Lemma $2(i)$, this gives a $(2(u-v), u)$ cover and hence the desired $(t, u)$ cover.

Consider now examples with $X^{\prime} \subseteq X, Y^{\prime} \subseteq Y,\left|X^{\prime}\right|=s-v,\left|Y^{\prime}\right|=s-w$ and $G$ having all edges except those of the form $\left(x^{\prime}, y^{\prime}\right)$. First take $v=0$ and $w=u-1$, then $|E|=s^{2}-(s-v)(s-w)=s(u-1)$ and $G$ has no $(t, u)$ cover. This shows that Theorem $2(\mathrm{i})$ is best possible. Now with general $v$ let $w=2 \dot{u}-t-1-v$. We make $0<v<s-w$ so $w<s-v$. We make $t<2(u-v)$ because we need $v \leqq w$ to keep the valency right. Also we make $2 v<u$ to keep $u \leqq t$. Thus $m=v+w=2 u-t-1$ and $|E|=s^{2}-(s-v)(s-w)=$ $(s-v)(2 u-t-1)+v^{2}$. There is no $(t, u)$ cover by Lemma 1 (iii) so Theorem 2(ii) is best possible. Finally to see Lemma 3 is best possible put $t=u$.

\section{The circuit problem.}

PROOF OF THEOREM 3. By suitably numbering the vertices of $X$ and $Y$, let $x_{1}, y_{1}, x_{2}, y_{2}, \cdots$ be a path of maximum possible length in $G$. This path must contain at least $v$ vertices $y_{i}$ which are adjacent to $x_{1}$, for otherwise we could make it longer. If $y_{j}$ is the last such $y_{i}$ then $x_{1}, y_{1}, x_{2}$, $y_{2}, \cdots, x_{j}, y_{j}, x_{1}$ is a circuit of length at least $2 v$. The example where $G$ consists of two separate complete bipartite graphs, each having $v$ vertices in one part and $s-v$ vertices in the other part, shows the result is best possible.

Proof of Theorem 4. We know by Theorem $2^{\prime}$ that $G$ has at least one matching. We will in fact show that any matching $\left(x_{1}, y_{1}\right),\left(x_{2}, y_{2}\right), \cdots$, $\left(x_{s}, y_{s}\right)$ of $G$ is "half" of a Hamiltonian circuit. Assume this is not the case. Adjoin as many edges as possible to $G$ without forming a Hamiltonian circuit containing the matching. This cannot make $G$ complete, so some vertex $y_{s}$ is not adjacent to $x_{1}$, say. If we adjoined $\left(x_{1}, y_{s}\right)$ we would get a Hamiltonian circuit containing the matching, so we may assume we have the path $x_{1}, y_{1}, x_{2}, y_{2}, \cdots, x_{s}, y_{s}$. For $1<i<s$ we cannot have both of the edges $\left(x_{1}, y_{i}\right)$ and $\left(x_{i}, y_{s}\right)$ in $G$ for otherwise we have the circuit

$$
x_{1}, y_{1}, x_{2}, y_{2}, \cdots, x_{i}, y_{s}, x_{s}, y_{s-1}, x_{s-1}, \cdots, y_{i+1}, x_{i+1}, y_{i}, x_{1} .
$$

Therefore the sum of the local degrees of $x_{1}$ and $y_{s}$ is at most $s$, and so $v \leqq \frac{1}{2} s$. The same is true for any vertex $y_{i}$ not adjacent to $x_{1}$. If $v^{\prime}=\left|\Gamma x_{1}\right|$, there are $s-v^{\prime}$ such $y_{i}$ and each has local degree $\leqq s-v^{\prime}$. Hence $|E| \leqq$ $\left(s-v^{\prime}\right)^{2}+s v^{\prime}$ and this implies $|E| \leqq(s-v) s+v^{2}$ because $v \leqq v^{\prime}$. The proof is 
now complete. The example where $G$ consists of all edges $\left(x_{i}, y_{j}\right)$ except those with $1 \leqq i \leqq v \leqq \frac{1}{2} s$ and $v<j \leqq s$ shows the theorem is best possible.

\section{REFERENCES}

1. Alan Brace, Some combinatorial cover theorems, Ph.D. Thesis, University of Western Australia, 1971.

2. C. Berge, Théorie des graphes et ses applications, Collection Univ. Math., II, Dunod, Paris, 1958; English transl., Methuen, London; Wiley, New York, 1962. MR 21 \#1608; 24 \#A2381.

3. D. R. Woodall, Sufficient conditions for circuits in graphs, Proc. London Math. Soc. (3) 24 (1972), 739-755.

School Information Sciences, Canberra College of Advanced Education, Canberra, australia

Department of Mathematics, University of Reading, Berkshire, United KINGDOM 